\title{
MOEA based Design of Decentralized Load-Frequency Controllers for Interconnected Power Systems with AC-DC Parallel Tie-lines and Considering Nonlinearities
}

\author{
S.Ganapathy \\ Department of Electrical Engineering \\ Annamalai University \\ Annamalainagar, Tamilnadu,India-608002
}

\author{
S.Velusami \\ Department of Electrical Engineering \\ Annamalai University \\ Annamalainagar, Tamilnadu,India-608002
}

\begin{abstract}
A new approach to the design of decentralised controllers, using Multi-Objective Evolutionary Algorithm (MOEA), for loadfrequency control of interconnected power systems with AC-DC parallel tie-lines and considering Governor Dead Band and Generation Rate Constraint nonlinearities, is proposed in this paper. The HVDC link is used as system interconnection in parallel with AC tie-line to effectively damp the frequency oscillations of AC system. The proposed controller satisfies two main objectives, namely, minimum Integral Squared Error of the system output and maximum closed loop stability of the system. Simulation studies are conducted on a two area interconnected power system with AC-DC parallel tie lines and considering nonlinearities. Results indicate that the proposed controller gives good dynamic responses and can guarantee the stability of the overall system in the presence of system nonlinearities and parameter changes.
\end{abstract}

\section{Categories and Subject Descriptors}

J.2 [Computer Applications]: Physical Sciences and Engineering - engineering-electrical-power system

\section{General Terms}

Algorithm, Design, Performance

\section{Keywords}

Power systems, Load-frequency control, Multi-Objective Evolutionary Algorithm, HVDC link, AC-DC tie-lines, Governor dead band, Generation rate constraint

\section{INTRODUCTION}

Load-frequency control (LFC) is important for large interconnected power systems, to keep the system frequency and the inter-area tie-power as near to the scheduled values as possible. Many control strategies have been proposed and investigated for LFC of power systems by several researchers over the past decades [1]. Majority of the works reported in the literature have been carried out with interconnected power systems considering the area interconnection with $\mathrm{AC}$ tie-lines.

However, there has been a tremendous growth of the HVDC transmission system. The main interest in HVDC technology is because they offer economic, environmental and performance advantages over the other alternatives. For example, it is found to be more economical to adopt HVDC for asynchronous interconnections, underground cable systems and long distance transmissions. HVDC offers a viable and cost effective means of transferring large blocks of power between the remote generating stations and the load network. Another advantage of the HVDC is that the DC power flow on the line is highly adjustable. Therefore, power flow oscillations in an AC system due to system disturbances can be effectively damped by controlling the DC power.

Because of the unique advantages of HVDC transmission systems, it has been applied widely in operating a DC link in parallel with an AC link interconnecting control areas to get an improved system dynamic performance with greater stability margins under small disturbances in the system. Only few researches have been carried out on LFC of interconnected power systems connected via HVDC link in parallel with AC link [2]. Therefore, this paper considers LFC of an interconnected power system with a DC tie-line in parallel with an AC tie-line. Incremental DC power flow is considered as an additional state variable in the LFC strategy.

Many investigations in the area of decentralized LFC of interconnected power systems have been reported in the past [1] and a number of control strategies have been proposed to achieve improved performance. However, proportional plus integral controllers are traditionally used for LFC of interconnected power systems because of their inherent simplicity, easy realization, robust and decentralized nature of the control strategy. But, in most cases, while designing the controllers, the mathematical model of the power system has been oversimplified by ignoring the simultaneous presence of important system nonlinearities such as Governor Dead Band (GDB) and Generation Rate Constraint (GRC). In the presence of nonlinearities, the dynamic response of the system is associated with large overshoots and longer settling times [3]. Hence, it becomes important to consider these system nonlinearities while designing the controllers.

The Integral Squared Error (ISE) criterion is used for obtaining the controller gain settings [4]. However, the frequency deviations and tie-line power deviations persist for a long duration even though zero steady state errors are ensured. The controller designed on the basis of Integral Squared Error criterion tends to show a rapid decrease in the large initial error. 
Hence, the response is fast and oscillatory. Thus, the system has poor relative stability. Hence, to obtain the decentralized controllers with improved stability margin, they are designed on the basis of Maximum Stability Margin (MSM) criterion using Lyapunov method. However, Controllers designed on the basis of MSM criterion do not posses the inherent good properties of the controller designed on the basis of ISE criterion except for improvement in stability [5]. Therefore, it is expected that an appropriate multi-objective control strategy will be able to give a better solution for this problem.

There are different intelligent controllers in the literature that have been used in isolated as well as interconnected power systems [6]. However, they have been mainly applied to LFC problems treated as single-objective optimization problems.

Hence, a new design of proportional plus integral controllers using Multi-Objective Evolutionary Algorithm (MOEA) is proposed in this work, for the decentralized load-frequency control of interconnected power systems with AC-DC tie-lines and considering system nonlinearities, to achieve a good dynamic response besides excellent closed loop stability of the system. The LFC problem is formulated as a Multi-Objective Optimization problem where ISE criterion and MSM criterion are treated as competing objectives. The proposed controller has been applied to an interconnected two-area thermal power system with AC-DC parallel tie-lines. Each area contains two thermal units with GDB and GRC nonlinearities.

\section{STATEMENT OF PROBLEM}

The proposed decentralized controller design is applied to an interconnected two-area thermal power system with AC-DC parallel tie-lines, as shown in Figure 1. Each area comprises of two units and includes GDB and GRC nonlinearities. Data for the system is taken from [2,9].

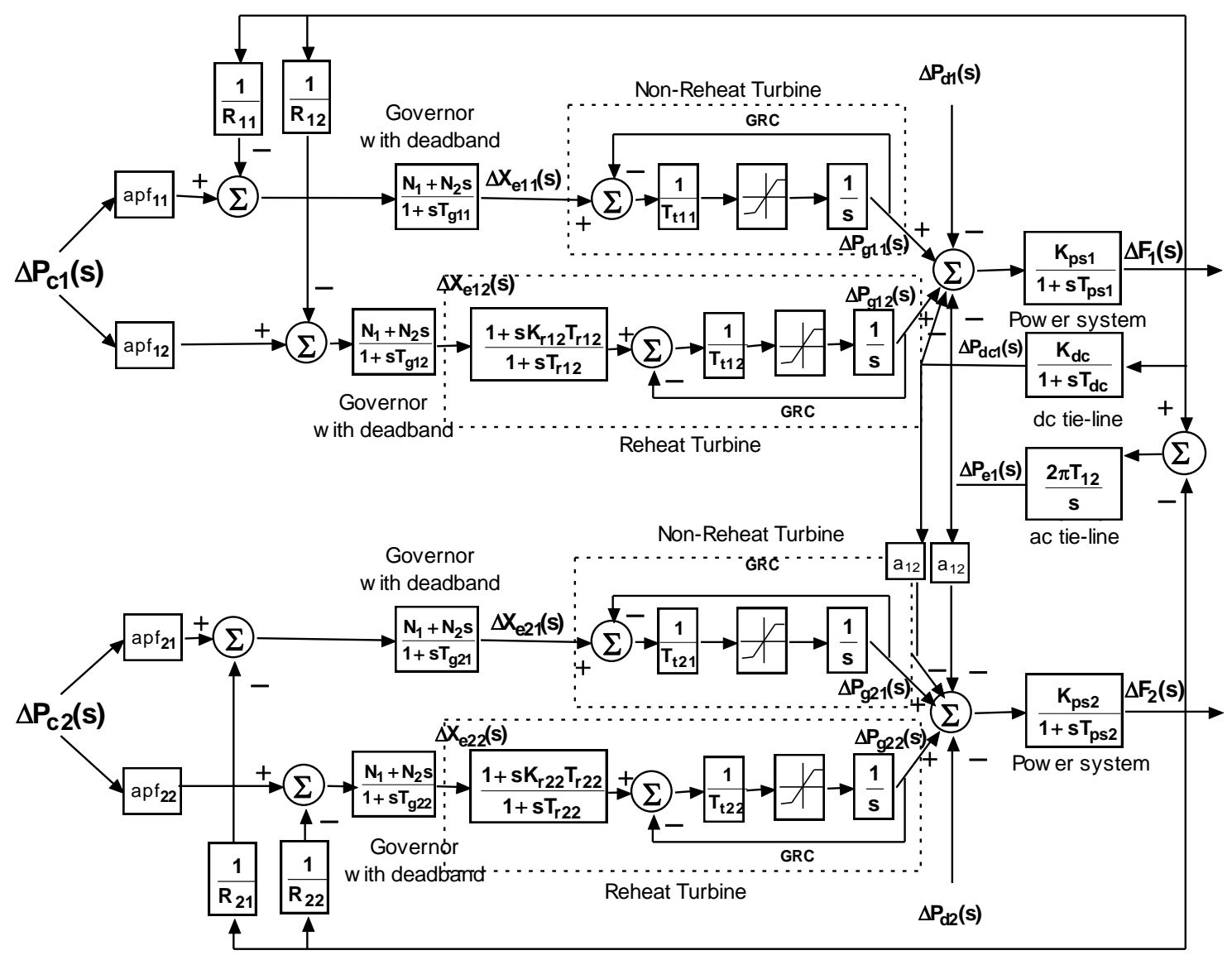

Figure 1. Block diagram representation of a two area interconnected thermal power system with AC-DC parallel tie-lines and considering nonlinearities.

GDB is defined as the total magnitude of a sustained speed change within which there is no change in valve position. A describing function approach is used to incorporate the GDB nonlinearity $[3,9]$.
In the real life situation, there is a maximum limit to the rate of change in generated power of a steam plant. It has been shown that dynamic responses of the system with the presence of GRC have larger overshoots and longer settling times, when compared 
to the system wherein GRC is neglected [3]. Moreover, if the parameters of the controller are not designed properly, the system may become unstable. A generation rate limitation of $3 \%$ per minute is considered for the thermal power plants, in this study.

The dynamic behaviour of the LFC system is described by the state space equation

$$
\dot{x}=A x+B u+\Gamma d
$$

$$
\begin{aligned}
& \boldsymbol{x}=\left[\Delta F_{1} \Delta P_{g 11} \Delta X^{\prime} \text { el1 } \Delta P_{g 12} \Delta P^{\prime} g 12 \Delta X^{\prime} e 12 \Delta P_{e l}\right. \\
& \left.\Delta P_{d c e 1} \Delta F_{2} \Delta P_{g 21} \Delta X_{e 21}^{\prime} \Delta P_{g 22} \Delta P_{g 22}^{\prime} \Delta X_{e 22}^{\prime}\right]^{\Gamma} \\
& \boldsymbol{u}=\left[\begin{array}{ll}
\Delta P_{c 1} & \Delta P_{c 2}
\end{array}\right]^{\mathrm{T}} \\
& \boldsymbol{d}=\left[\begin{array}{ll}
\Delta P_{d 1} & \Delta P_{d 2}
\end{array}\right]^{\mathrm{T}}
\end{aligned}
$$

are the state, control and disturbance vectors and $\boldsymbol{A}, \boldsymbol{B}$ and $\boldsymbol{\Gamma}$ are respectively system state matrix, control input matrix and disturbance input matrix of appropriate dimensions. The corresponding co-efficient matrices are obtained using the nominal system parameter values given in appendix. A step load disturbance of $1 \%$ has been considered as a disturbance in the system.

It is known that, by incorporating an integral controller, the steady state requirements can be achieved.

In order to introduce integral function in the controller, the system equation (1) is augmented with new state variables defined as the integral of ACE $\left(\int v_{i} d t\right), i=1,2$.

The augmented system of the order $(2+n)$ may be described as

$$
\begin{gathered}
\dot{\overline{\boldsymbol{x}}}=\overline{\boldsymbol{A}} \overline{\boldsymbol{x}}+\overline{\boldsymbol{B}} \boldsymbol{u}+\overline{\boldsymbol{\Gamma}} \boldsymbol{d} \\
\text { Where } \overline{\boldsymbol{x}}=\left[\begin{array}{c}
\int \boldsymbol{v} \boldsymbol{d} \boldsymbol{t} \\
\boldsymbol{x}
\end{array}\right] \begin{array}{l}
/ N \\
/ n
\end{array} \\
\overline{\boldsymbol{A}}=\left[\begin{array}{ll}
\boldsymbol{0} & \boldsymbol{C} \\
\boldsymbol{0} & \boldsymbol{A}
\end{array}\right] ; \overline{\boldsymbol{B}}=\left[\begin{array}{l}
\boldsymbol{0} \\
\boldsymbol{B}
\end{array}\right] \text { and } \overline{\boldsymbol{\Gamma}}=\left[\begin{array}{c}
\boldsymbol{0} \\
\boldsymbol{\Gamma}
\end{array}\right]
\end{gathered}
$$

The decentralized feedback control law may be written in-terms of $v_{i}$ as [7]

$$
u_{i}=-k_{i 1} \int v_{i} d t-k_{i 2} v_{i}, i=1.2 \ldots . ., N
$$

where $\boldsymbol{k}_{\boldsymbol{i}}^{\boldsymbol{T}}=\left[\begin{array}{ll}\boldsymbol{k}_{\boldsymbol{i} \boldsymbol{1}} & \boldsymbol{k}_{\boldsymbol{i} 2}\end{array}\right]$ is a 2-dimensional integral and proportional feedback gain vector. This design assumes that, the two area interconnected power system consists of 2 - identical areas. Therefore, the decentralized integral feedback gains $\left(k_{11}=k_{21}=k_{I}\right)$ and the decentralized proportional controller feedback gains $\left(k_{12}=k_{22}=k_{P}\right)$ of the 2 - identical areas are assumed to be equal.

\section{DESIGN OF DECENTRALIZED CONTROLLER USING ISE CRITERION}

The objective is to obtain the optimum values of the controller parameters that minimize the performance index,

$$
J_{i}=\int_{0}^{\mathrm{t}}\left(\boldsymbol{x}_{e i}^{\boldsymbol{T}} \boldsymbol{W}_{i} \boldsymbol{x}_{e i}\right) \mathrm{dt}, \quad i=1,2, \ldots, N
$$

where

$\boldsymbol{W}_{i}=\operatorname{diag}\left\{w_{i 1} w_{i 2} w_{i 3}\right\}$ and $\boldsymbol{x}_{e i}{ }^{T}=\left[\begin{array}{ll}\Delta f_{i} \Delta p_{e i}, \Delta p_{d c e l}\end{array}\right]$

$w_{i 1}, w_{i 2}$ and $w_{i 3}$ are weighting factors for the frequency deviation, AC tie-line power deviation and DC tie-line power deviation respectively of area $\mathrm{i}$ and are chosen as unity. The decentralized proportional plus integral controller gains using ISE criterion are designed as discussed in [3] and the values obtained are $k_{P}=0.79$ and $k_{I}=0.37$.

\section{DESIGN OF DECENTRALIZED CONTROLLER USING MSM CRITERION}

The controller designed on the basis of Integral Squared Error criterion tends to show a rapid decrease in the large initial error. Hence, the response is fast and oscillatory. Thus, the system has poor relative stability [8]. Therefore, the design of proportional plus integral controller with improved stability using MSM criterion by Lyapunov method [9] is discussed in this section.

The stability index to be minimized is

$$
\stackrel{\bullet}{x}=\hat{A} \bar{x}+\bar{\Gamma} \bar{d}
$$

The stability index $\boldsymbol{\eta}$ to be minimized can be written as

$$
\eta=\bar{x}^{T} P \bar{x}
$$

Where $\boldsymbol{P}$ is a symmetric positive definite matrix obtained from the solution of

$$
\hat{\boldsymbol{A} P}+\boldsymbol{P} \hat{\boldsymbol{A}}=-\boldsymbol{Q}
$$

where $\boldsymbol{Q}$ is a positive semi-definite matrix and $\hat{\mathrm{A}}$ is augmented system matrix. The weighting matrix $\boldsymbol{Q}$ is $\boldsymbol{Q}=\operatorname{diag}\{0,0,1,100,0,100,0,0,1,1,1,100,0,100,0,0\}$.The weight coefficients corresponding to the output variables $\Delta P_{g 11}, \Delta P_{g 12,} \Delta P_{g 21}$ and $\Delta P_{g 22}$ are specified to relatively larger values in order to prevent the excessive control action when GRC is taken into account.

The proportional controller feedback gain $k_{P}$, corresponding to minimum value of stability index $\boldsymbol{\eta}$, is obtained using the MSM criterion by plotting the stability curve for various values of $k_{P}{ }_{P}$ against the stability index $\boldsymbol{\eta}$ [9]. The integral feedback gain $k_{I}^{\prime}$ is treated as zero throughout in this design. From the stability curve, the optimal proportional controller feedback gain 
$k_{P}^{\prime}=-0.14$ is obtained. Next, the stability curve for various values of $k_{I}^{\prime}$ is obtained by simulating the closed loop system and keeping $k_{P}^{\prime}=k_{P}^{\prime}($ opt $)$. From the curve, the optimal integral controller feedback gain $k_{I}^{\prime}=1.1$ is obtained.

\section{DESIGN OF PROPOSED DECENTRALIZED CONTROLLER}

Controllers designed on the basis of MSM criterion do not posses the inherent good properties of the controller designed on the basis of ISE criterion except for improvement in stability. Therefore, a new controller design needs to be developed based on a compromise between the ISE design criterion and MSM design criterion in order to obtain satisfactory closed loop system performance and stability [5].

An attempt has been made in this section to design a decentralized controller using Multi-Objective Evolutionary Algorithm.

\subsection{Multi-Objective Evolutionary Algorithm}

Multi-objective optimization methods deal with finding optimal solutions to problems having multiple objectives. These objectives often conflict each other so that improving one of them will deteriorate another objective function. Therefore, the solution to a Multi-objective optimization problem is normally not a single value but instead a set of values called the "ParetoOptimal Set" [10]. No solution from this set of optimal solution can be said to be better than another solution. This procedure is practical because the user gets an opportunity to investigate a number of other trade-off solutions before choosing one particular optimal solution.

Evolutionary Algorithms (EAs) are a natural choice for solving multi-criterion optimization problems because of their population-based nature. A number of Pareto-optimal solutions can, in principle, be captured in an EA population, thereby allowing a user to find multiple Pareto-optimal solutions in one simulation run. Different approaches of MOEA have been used by different researchers for multi-objective optimization, each one having its merits and demerits [11]. Among various MOEAs, $\varepsilon$ - MOEA has shown the best performance.

Hence, in this study, a steady state Multi-Objective Evolutionary Algorithm based on $\varepsilon$-dominance concept is used [12].

Here, two populations (EA and archive) are evolved simultaneously and independently. Using one solution each from both populations, two off-spring solutions are created through mating. Each off-spring is then used to update both parent and archive populations. The archive population is based on the $\varepsilon$-dominance whereas a usual dominance concept is used to update the present population. The final archive members after a specified number of iterations are reported as the obtained solutions [12]. The algorithm for $\varepsilon-$ MOEA[13] is given below:

Step1: Randomly generate initial pool.

Step2: Sort by domination, and set first front as archive.

Step3: Generate a new individual by choosing the parents from the population and archive. (a) Choose two individuals from population.

(b) Choose dominating solution, if dominates; choose random one, otherwise.

(c) Choose one individual from archive.

(d) Perform crossover and mutation.

Step4: Update archive.

(a) Replace $\varepsilon$-dominated individual(s) in the archive with new individual, if new individual $\varepsilon$-dominates archive member(s).

(b) Leave dominating member, if there are more than one archive members in the same grid.

(c) Add new individual, if archive members do not dominate new individual.

Step5: Update population.

(a) Replace dominating individual(s) with new individual.

(b) Replace randomly selected population member with new individual, if there is no population member which dominates the new individual.

Step6: Check termination criteria.

\subsection{Design of Proposed Decentralized Controller using MOEA}

An attempt has been made in this section, to apply MOEA to the LFC problem with ISE criterion and MSM criterion as conflicting objectives.

The LFC problem can be formulated as

Minimize

$$
\begin{aligned}
& f_{1}(\mathbf{X})=f_{1}\left(\mathrm{x}_{1}, \mathrm{x}_{2}\right)=f_{1}\left(k_{P m}, k_{I m}\right)=\boldsymbol{J}_{\boldsymbol{I}} \\
& f_{2}(\mathbf{X})=f_{2}\left(\mathrm{x}_{1}, \mathrm{x}_{2}\right)=f_{2}\left(k_{P m}, k_{I m}\right)=\boldsymbol{\eta}
\end{aligned}
$$

Subject to

$$
\begin{aligned}
& k_{P m}{ }^{(L)} \leq k_{P m} \leq k_{P m}(U) \\
& k_{I m}(L) \leq k_{I m} \leq k_{I m}(U)
\end{aligned}
$$

The proportional controller feedback gains obtained by ISE and MSM criteria, namely $k_{P}=0.79$ and $k_{P}{ }_{P}=-0.14$, are treated as the upper and lower bounds for the decision variable $k_{P m}$ in the MOEA. Similarly, the integral controller feedback gains obtained by MSM criterion and ISE criterion, namely, $k_{I}^{\prime}=1.1$ and $k_{I}=0.37$, are treated as the upper and lower bounds for the decision variable $k_{I m}$. The proposed controller feedback gains are obtained as $k_{P m}=0.14$ and $k_{I m}=1.0$ using MOEA. This design ensures that, the controller feedback gains will always be within the ranges of the gains obtained from the ISE criterion and the MSM criterion. Therefore, the controller will guarantee the stability. Further the controller possesses improved stability when compared to the controller obtained using ISE criterion. The overall performance of these controllers will be better than that of the controller designed on the basis of MSM criterion. The choice of $\varepsilon$-MOEA parameters was done according to 
general guidelines available in the literature. A population size of 100, the real-parameter Simulated Binary Cross-over (SBX) recombination operator with a crossover probability of 1 and a distribution index of 15 for crossover, and a polynomial mutation operator with a mutation probability of $1 / n(n=$ number of decision variables) and a distribution index of 20 for mutation, have been used. The recommended values of $\varepsilon_{1}=0.05$ and $\varepsilon_{2}=0.05$ are found to be robust enough and are used in our study.

\section{SIMULATION RESULTS}

The decentralized controller with output feedback is designed using MOEA with multiple objectives, namely the ISE criterion and MSM criterion, and implemented in the interconnected twoarea thermal power system. The system is simulated with the proposed controller for 0.01 p.u.MW step load change in area 1 and the corresponding frequency deviation $\Delta f$ and tie -line power deviation $\Delta P_{e}$ are plotted with respect to time. For easy comparison, the responses of $\Delta f$ and $\Delta \mathrm{P}_{\mathrm{e}}$ of the system are shown along with the responses obtained with the optimal decentralized proportional plus integral controller designed based on ISE criterion in Figure 2.

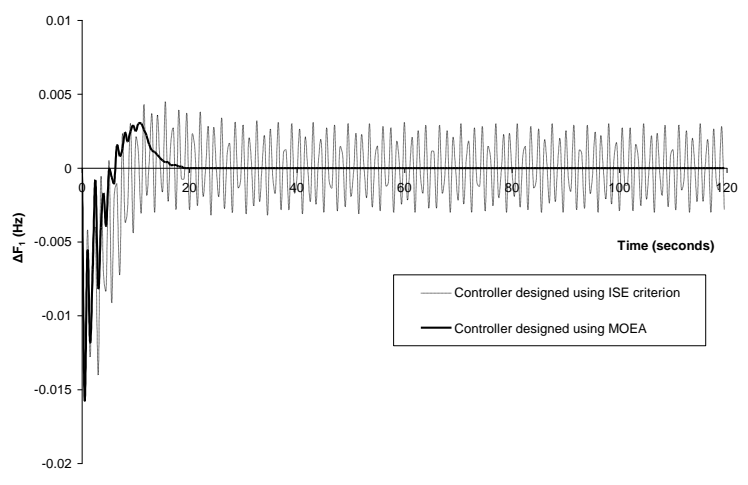

(a)Frequency deviation of area 1

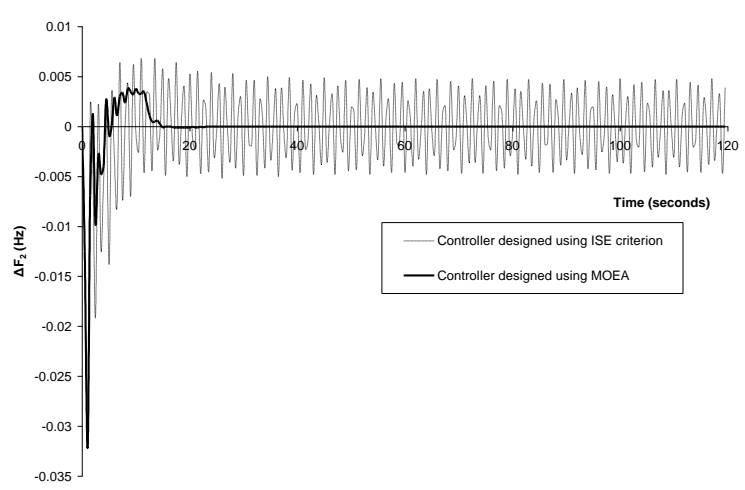

(b) Frequency deviation of area 2

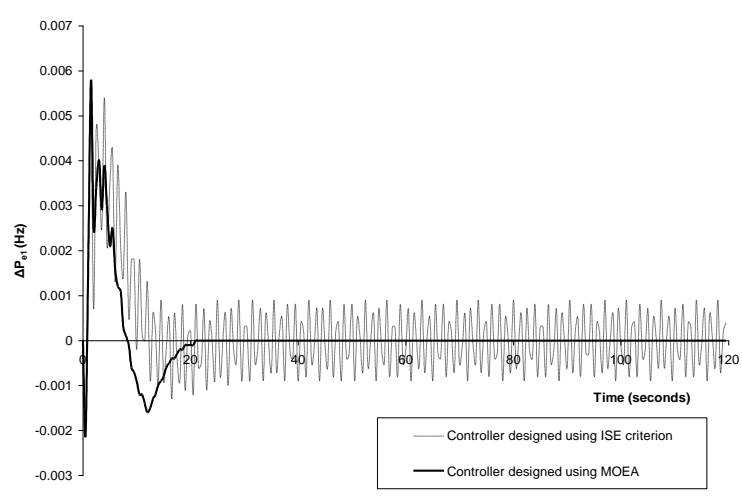

(c) AC Tie-line power deviation of area 1

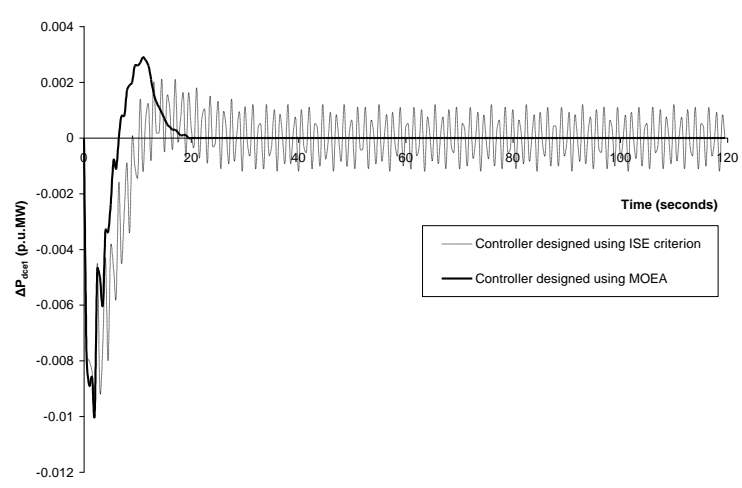

(d) DC Tie-line power deviation of area 1

Figure 2. Frequency deviations and tie-line power deviation for 0.01 p.u.MW step load change in area 1 with MOEA based decentralized controller.

The Gain values and cost function values of the various controllers are given in Table 1.

Table 1. Comparison of cost function values

\begin{tabular}{|c|c|c|}
\hline $\begin{array}{c}\text { Type of proportional } \\
\text { plus integral controller }\end{array}$ & $\begin{array}{c}\text { Feedback } \\
\text { gains }\end{array}$ & $\begin{array}{c}\text { cost } \\
\text { function } \\
\text { value }\end{array}$ \\
\hline $\begin{array}{c}\text { Controller designed } \\
\text { using ISE criterion }\end{array}$ & $k_{P}=0.79$ & 0.4945 \\
\hline $\begin{array}{c}\text { Controller designed } \\
\text { using MOEA }\end{array}$ & $k_{P m}=0.37$ & 0.14 \\
$k_{I \mathrm{~m}}=1.0$ & 0.2122 \\
\hline
\end{tabular}


It is observed from Figure 2 and Table 1 that the proposed controller has fast response and excellent transient performance, with reduction in cost function value.

Any further improvement in one of the design objectives will lead to degradation in the other objective.

\section{CONCLUSION}

A Multi-Objective Evolutionary Algorithm based design of decentralized controllers for load-frequency control of interconnected power systems, with AC-DC parallel tie-lines and considering GDB and GRC nonlinearities, is presented. The proposed controller design has been successfully applied to an interconnected two-area thermal power system with two units in each area taking into account the system nonlinearities. Simulation results reveal that the designed controller is very effective in suppressing the frequency and tie-line power oscillations while simultaneously improving the system stability.

\section{ACKNOWLEDGMENTS}

The authors wish to thank the authorities of Annamalai University, Annamalainagar, Tamilnadu, India for the facilities provided to prepare this paper.

\section{REFERENCES}

[1] Shayeghi, H., Shayanfar, H. A., and Jalili, A. 2009. Load frequency control strategies: A state-of-the-art survey for the researcher. Energy Conv. Management. 50, 2 (Feb.2009), 344-353.

[2] Kumar, P. and Ibraheem, 1998. Dynamic performance evaluation of 2-area interconnected power systems: a comparative study. J. Institution of Engineers (India). 78, (Mar. 1998), 199-209.

[3] Nanda, J., Kothari, M.L., and Satsangi, P.S. 1983. Automatic Generation Control of Reheat Thermal system considering Generation Rate Constraint and Governor Deadband. Journal of Institution of Engineers (India). 63, 6 (June, 1983),245-252.
[4] Elgerd, O. E., and Fosha, C. E. 1970. Optimum megawatt-frequency control of multi area electric energy systems. IEEE Trans. Power App. Syst. 89, 2 (Apr. 1970),556-563.

[5] Chidambaram, I. A., and Velusami, S. 2005. Design of decentralized biased controllers for load-frequency control of interconnected power systems. Elect. Power Compon. Syst. 33 (Dec.2005), 1313-1331.

[6] Mathur, H. D., and Ghosh, S. 2006.A comprehensive analysis of intelligent controllers for load frequency control. In Proc. IEEE Power India Conference(New Delhi,India, May,2006) 853-857.

[7] Ramar, K., and Velusami S. 1989. Design of decentralized load-frequency controllers using pole placement technique. Elect. Mach. Power Syst. 16 (Jan.1989), 193-207.

[8] Ogatta, K. 1970. Modern control engineering. PrenticeHall, New Jersey.

[9] Tripathy, S. C., Hope, G. S., and Malik, O. P. 1982. Optimisation of load-frequency control parameters for power systems with reheat steam turbines and governor dead band nonlinearity. IEE Proc. 129, 1(Jan. 1982) 10-11.

[10] Ghosh,A., and Dehuri, S., 2004. Evolutionary algorithms for multi-criterion optimization: a survey. Int. J. Comp. Information Sciences. 2, 1 (Apr. 2004),38-57.

[11] Deb, K. 2004. Multi-objective optimization using evolutionary algorithms. John Wiley, UK.

[12] Deb, K., Mohan, M., and Mishra, S. 2003. A fast multiobjective evolutionary algorithm for finding well-spread pareto-optimal solutions. kanGAL Technical Report No. 2003002. Kanpur Genetic Algorithms Laboratory, Indian Institute of Technology, Kanpur, India. (Feb.2003), 1-18.

[13] Shin, S. -Y., Lee., I. -H., Zhang, B. -T., 2006. Micro array probe design using $\varepsilon$-multi-objective evolutionary algorithms with thermodynamic criteria. Springer Berlin. LNCS (EvoBIO2006) 3907, 184-195. 